\title{
Periodic respiration in erect posture in Shy-Drager syndrome
}

\author{
S. CHOKR OVERTY, J. T. SHAR P, A N D K. D. BARR O N \\ From the Neurology Service, Neurology Research Laboratory, and Pulmonary Section, \\ Medical Service, Veterans Administration Hospital, Hines, Illinois, and Department of Neurology, \\ Albany Medical College, Albany, New York, USA
}

SUMMARY Tilt-table polygraphic study in four patients with Shy-Drager syndrome demonstrated periodic apnoea in the erect posture. In one patient reduced hypercapneic ventilatory response and necropsy findings of neuronal loss and astrocytosis in the pontine tegmentum suggested dysfunctional respiratory neurones in the brainstem. One patient had Cheyne-Stokes respiration during the late stage of the illness.

Postural changes of respiration in normal individuals were described by Turner (1927), Hamilton et al. (1932), Main (1937), McMichael (1938), and Franseen and Hellebrandt (1943). These changes consist of increase in the rate of respiration and ventilation, and decrease of alveolar $\mathrm{CO}_{2}$. However, little attention has been paid to the postural responses of respiration in primary orthostatic hypotension. Verel (1951) described three patients with primary orthostatic hypotension in whom the rate and depth of respiration on standing increased as the blood pressure fell. We report four patients with the Shy-Drager variant of primary orthostatic hypotension who demonstrated a striking periodicity in breathing during head-up tilt.

\section{Case reports}

Cases 1 and 2 have been published previously (Chokroverty et al., 1969) as cases 2 and 3 respectively.

\section{CASE 1}

In April 1967, a 52 year old man was admitted to the Neurology Service for investigation of syncopal episodes on standing, urinary frequency and incontinence of four years' duration, and impotence for 10 years.

On examination, the average readings of blood pressure and pulse rate in lying, sitting, and standing positions were as follows: lying, $160 / 90 \mathrm{mmHg}$

Address for reprint requests: Dr S. Chokroverty, PO Box 127, Hines, Illinois 60141, USA.

Accepted 30 May 1978 and 84 per min; sitting for two minutes, $88 / 68$ $\mathrm{mmHg}$ and 84 per min; standing for two minutes, $0 / 0 \mathrm{mmHg}$ and 84 per min. On neurological examination, he had brisk but symmetrical muscle stretch reflexes, absent superficial abdominal and cremasteric reflexes, and extensor plantar responses bilaterally.

In December 1968, additional neurological findings consisted of slight impairment of recent memory and an expressionless face. $\mathrm{He}$ was receiving 9-alpha-fluorohydrocortisone $1.2 \mathrm{mg} \mathrm{a}$ day.

In May 1969 he had pneumonia and generalised seizures, and he died on 14 May 1969.

On postmortem examination bronchopneumonia, mild cardiomegaly, passive congestion of the liver, and chronic cystitis were present. The brain weighed $1430 \mathrm{~g}$. Macroscopically, coronal slices through the cerebral hemispheres, basal ganglia, mesencephalon, and cerebellum were normal except for moderate, symmetrical dilatation of the cerebral ventricular system and the fourth ventricle. Transverse sections through the brainstem and cerebellum were noteworthy only for an impression of slight atrophy of the pons.

Blocks of the frontal, parietal, temporal, and occipital lobes, basal ganglia, thalamus, midbrain, pons, medulla, cerebellum, spinal cord, peripheral nerves, and skeletal muscle were embedded in paraffin, and stained with haematoxylin-eosin, Weil, Holzer, Nissl (cresyl violet), and Bodian techniques. There were no noteworthy abnormalities of the cerebral cortex and white matter. The putamen had a spongiform appearance and 
showed a marked loss of nerve cells accompanied by reactive gliosis. There was striking pallor of the striate bundles with myelin sheath stains. These alterations were most marked in the lateral half or two-thirds of this structure. Sections through pallidum, hypothalamus, and thalamus did not disclose abnormality. Mild spongiform changes with astrogliosis were noted in the claustrum. There were marked losses of pigment and nerve cells, and moderately severe reactive gliosis in the substantia nigra. Severe gliosis was evident in the inferior colliculi and the periaqueductal region. There was no abnormality of the pyramidal tracts or the superior cerebellar peduncle. Within the pontine tegmentum there was neuronal loss and diffuse astrocytosis (Fig. 1) while the medulla showed marked gliosis of the inferior olivary nuclei. The dorsal vagal nuclei exhibited neuronal loss and a few reactive astrocytes. In the cerebellum there was a mild to moderate, diffuse loss of Purkinje cells, and a marked proliferation of Bergmann glia. Bodian stains disclosed numerous axonal "torpedoes" in the granular cell layer while pericellular "baskets" were well-stained about spaces empty of Purkinje cells. The medullary cores of folia were pallid in Weil preparations. There was cell loss in the intermediolateral column of the thoracic spinal cord in addition to mild to moderate loss of neurones from the anterior horn of the spinal cord at all levels. Many axonal spheroids occurred within the cervical and lumbosacral enlargements. Skeletal muscle contained numerous atrophic bundles of muscle fibres adjacent to well-preserved fascicles, an appearance indicative of neurogenic atrophy. Sections of nerves of the upper and lower limbs and of the vagus nerve did not exhibit

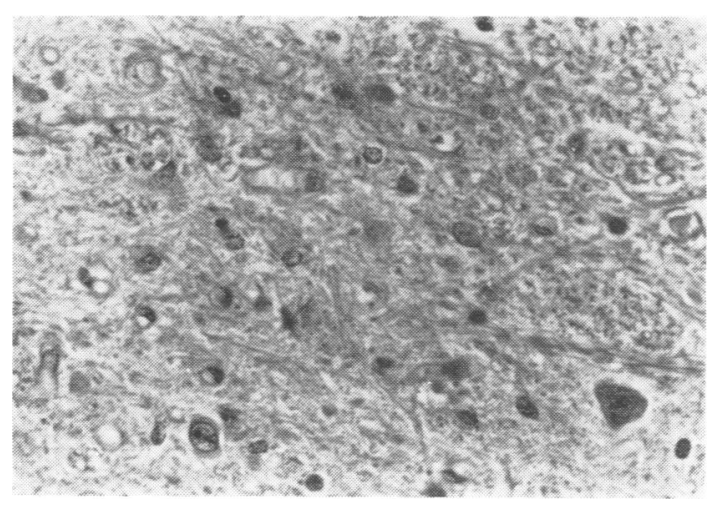

Fig. 1 Astrocytosis and neuronal loss in pontine tegmentum in the region of the reticular formation. Haematoxylin-eosin, original magnification $\times 640$. definite abnormality. The carotid sinus contained nerve fibres and had no unusual histological features.

\section{CASE 2}

A 55 year old man was admitted on 11 July 1967 with a two year history of "black-out spells" in the upright position and lack of sweating in the legs.

His blood pressure and pulse rate were as follows: lying $124 / 80 \mathrm{mmHg}$ and 70 per min; sitting for two minutes, $80 / 50 \mathrm{mmHg}$ and 82 per min; standing for four minutes, $0 / 0 \mathrm{mmHg}$ and 100 per min. Neurological examination showed evidence of mild cerebellar dysfunction in the upper limbs and ataxia of gait.

His cerebellar signs continued to progress and, in September 1968, he required a cane for walking because of marked ataxia of gait. His heart rate now did not show any postural variation. He died of bronchopneumonia in August 1971.

General postmortem examination revealed bronchopneumonia, pulmonary fibrosis, right ventricular hypertrophy, moderate coronary atherosclerosis, chronic passive congestion of the liver, kidneys, and spleen, leiomyoma of the stomach $30 \mathrm{~mm}$ above the pyloric antrum measuring $10 \times 15 \mathrm{~mm}$, and focal haemorrhages in the colon and terminal ileum. Macroscopic examination of the brain was remarkable only in that the pons appeared atrophic. The spinal cord was not removed at necropsy.

Microscopic examination of the cerebral hemispheres, basal ganglia, and thalamus did not show any significant abnormalities. The substantia nigra had a normal histological appearance. The periaqueductal region and oculomotor nuclei were normal. The cerebellum showed diffuse loss of Purkinje and granule cells. The cerebellar white matter was demyelinated and gliotic. The dentate nuclei showed nerve cell loss. The pontine nuclei were atrophied, and there was demyelination and gliosis of the pontocerebellar fibre tracts. In the medulla, there was neuronal loss and diffuse astrocytosis in the olivary nuclei bilaterally.

\section{CASE 3}

A 57 year old man was admitted to the Neurology Service on 30 September 1969 with a four month history of fainting spells and "dizziness" in the upright position, nocturia, and urinary frequency.

His supine blood pressure was $140 / 77 \mathrm{mmHg}$ and pulse rate 80 beats per minute. On standing for 2-3 minutes, blood pressure fell to $80 / 50$ $\mathrm{mmHg}$ and the pulse rate increased to 90 per min. Pertinent neurological findings consisted of widebased gait, terminal dysmetria on finger-nose and 
heel-shin tests, and decreased muscle stretch reflexes.

Results of laboratory tests were all normal except for a spastic neurogenic bladder (cystometrogram) and an abnormal breathing pattern accompanied by orthostatic fall of blood pressure demonstrated by polygraphic study. He was discharged on 14 November 1969, on 9-alphafluorohydrocortisone $0.4 \mathrm{mg}$ daily.

During his last admission in 1972, in addition to cerebellar dysfunction as noted above, he had an expressionless facies and cogwheel rigidity in both hands. He died in a nursing home on 5 December 1972.

CASE 4

A 49 year old man was admitted to the Neurology Service on 8 March 1976, with the complaints of impotence, urinary incontinence and frequency for three and a half years and "black-out spells" in the upright position, progressive loss of balance, slurring of speech, and lack of sweating for one year.

On examination, he had a supine blood pressure of $140 / 70 \mathrm{mmHg}$ and pulse rate of 80 beats per minute. On standing for 2-3 minutes, the blood pressure fell to $70 / 50 \mathrm{mmHg}$, but the pulse rate remained fixed. Neurological examination revealed a right Horner's syndrome, horizontal and downbeat nystagmus, scanning speech, truncal ataxia, broad-based ataxic gait, dysmetria, dysdiodochokinesis and terminal tremor on finger-nose and heel-shin tests. Muscle strength was good in all extremities. Muscle stretch reflexes were hyperactive, and plantar responses were extensor bilaterally. Sensation was normal.

Laboratory data were all normal except for the following: evidence of a hypotonic neurogenic bladder by cystometrogram; diffuse decrease of thermal sweating, marked below the level of the eighth thoracic segment; abnormalities of pulmonary tests, and polygraphic tracings of respiratory rate and rhythm.

He was discharged on 27 March 1976 on 9alpha-fluorohydrocortisone $0.5 \mathrm{mg}$ daily. $\mathrm{He}$ was readmitted on 19 July 1976, because of bronchopneumonia. While in the hospital, he had repeated episodes of respiratory tract infection, postural fainting, and hypotension. Neurological examination showed impairment of memory, judgment, and orientation in addition to the previously described findings. Despite treatment with antibiotics, he remained febrile, and died on 18 October 1976. Permission for postmortem examination was refused.

\section{Special investigations}

\section{METHOD}

Tilt-table polygraphic study

We have made several daytime simultaneous recordings of electroencephalogram (EEG), electromyogram (EMG) of the intercostal muscles, electrocardiogram (ECG), and abdominal breathing in all four patients on an electrically powered tilt-table. The studies were done with a Grass model 7 polygraph in cases 1 and 2 and a Grass 16-channel EEG model 6 in cases 3 and 4. Silver disc surface electrodes and the International 1020 electrode placement system were used for EEG recording. Intercostal EMGs were obtained by placing surface or needle electrodes in the right seventh or eighth intercostal space in the anterior or midaxillary line. One channel of the equipment was employed for recording a continuous lead I (upper precordial) ECG tracing. Respiration was recorded with a periabdominal pneumograph using a Grass preamplifier 7P1 or Grass model 6A1 low level DC amplifier and Grass volumetric pressure transducer PT5A. Blood pressure was measured every minute by auscultation of the brachial artery with a standard sphygmomanometer. The tracings were obtained at $0^{\circ}$ and $30^{\circ}$ tilt in cases 1 and 2 , and at $0^{\circ}, 30^{\circ}$, and $60^{\circ}$ tilt in cases 3 and 4 . All patients had been on treatment with 9-alphafluorohydrocortisone during this study.

\section{Pulmonary function tests}

These were performed in cases 1, 2, and 4 in both supine and erect $\left(30^{\circ}\right.$ head-up) positions. We measured the respiratory rate, tidal volume, vital capacity, minute ventilation, and alveolar ventilation by the standard technique. Arterial oxygen tension $\left(\mathrm{PaO}_{2}\right)$ and carbon dioxide tension $\left(\mathrm{PaCO}_{2}\right)$ were determined by withdrawing blood from the right radial artery with a Riley needle. Hypercapneic ventilatory response was measured in cases 1 and 4 in erect and supine positions by having the patients breathe $5-7.5 \% \mathrm{CO}_{2}$ in room air for five minutes, and determining the $\mathrm{PaCO}_{2}$ and minute ventilation.

\section{RESULTS}

Tilt-table polygraphic study

All measurements were normal at $0^{\circ}$ tilt except for supine hypertension in cases 1,3 , and 4 . When the table was tilted to $30^{\circ}$ (cases 1 and 2) or $60^{\circ}$ (cases 3 and 4), there were 5-10 episodes of apnoea or ineffective breathing or both of $8-22$ seconds duration accompanied by fall of blood pressure. The patients were kept in the tilted positions for approximately five minutes. Generally the respira- 
tion ceased in expiration. Intercostal EMG was silent during apnoea, and showed marked reduction of the interference pattern during ineffective breathing. The EEG demonstrated diminution or slight slowing of the background rhythm during apnoea or abortive respiration. Heart rate increased slightly in the erect posture in cases 3 and 4. Representative samples of polygraphic tracings in supine and tilted positions are shown in Figs. 2 and 3.

Five months after the initial study and two months before his death, a repeat polygraphic investigation in case 4 showed mild diffuse slowing
$(7 \mathrm{~Hz})$ of the EEG background rhythm. Furthermore, he showed Cheyne-Stokes respiration on this occasion both at $0^{\circ}$ and $30^{\circ}$ tilt (Fig. 4). The apnoeic periods were longer and hyperpneic periods were shorter in the erect position.

\section{Pulmonary function tests}

The Table lists the pulmonary function data. In the erect position, tidal volume increased slightly but the rate of respiration decreased. In cases 1 and 2, there was no significant change in $\mathrm{PaO}_{2}$ or $\mathrm{PaCO}_{2}$ in the erect position. Case 4 showed increased $\mathrm{PaO}_{2}$ and decreased $\mathrm{PaCO}_{2}$ in the upright

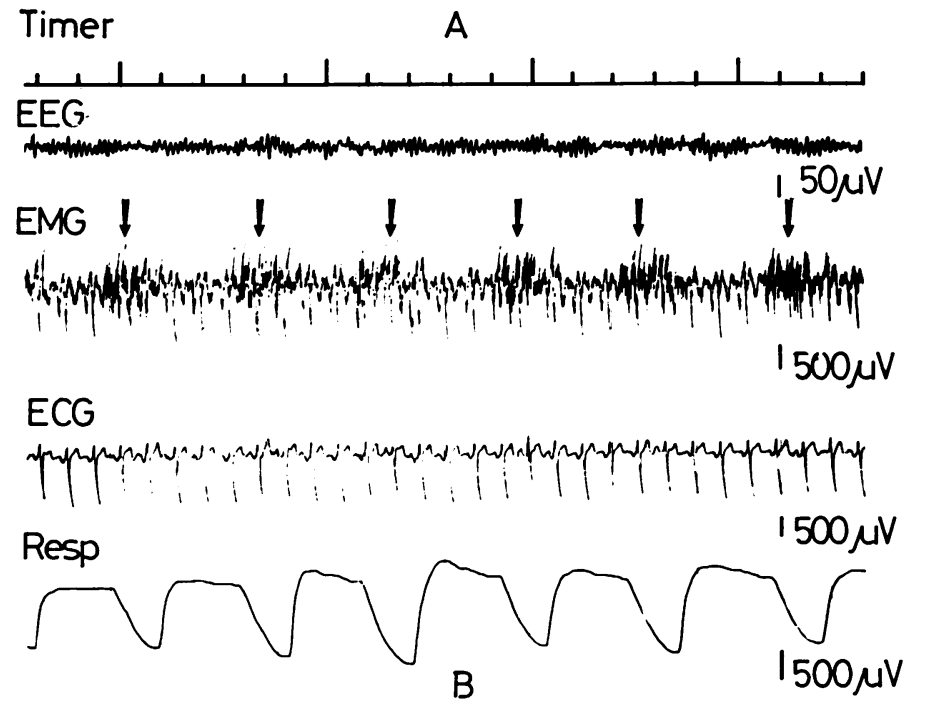

Timer

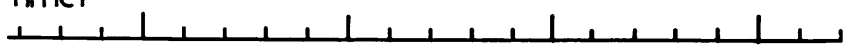

EEG

Den

EMG $\downarrow \downarrow \downarrow 150 \mu \mathrm{V}$

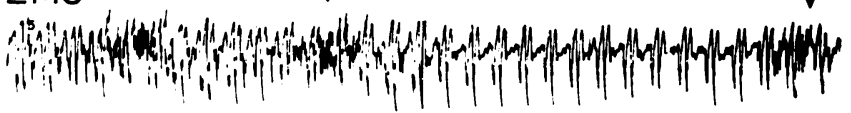

ECG

$1500 \mu \mathrm{VV}$

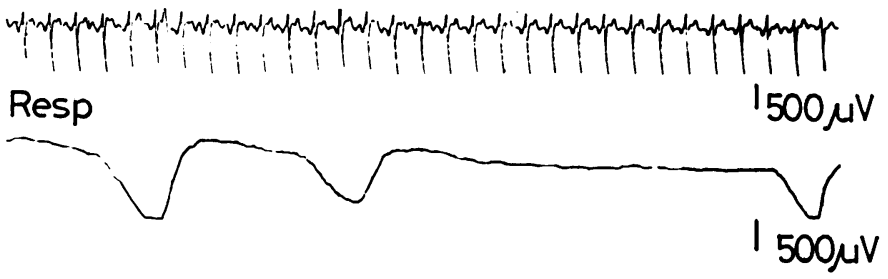

Fig. 2 (A) EEG $\left(P_{3}-A_{1}\right)$, intercostal $E M G$ (arrow indicates inspiratory activity), ECG, and Resp (abdominal respiration; downward deflection indicates inspiration) in case 1 in supine position. Timer: 1 second. (B) Same patient during head-up tilt. Note apnoeic period of 10 seconds. Timer: 1 second. 
Timer

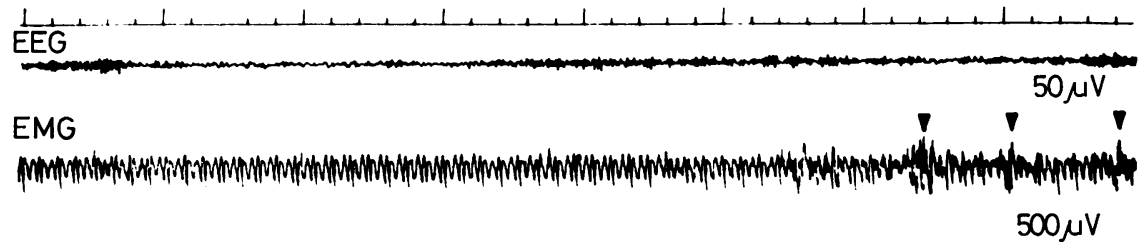

Resp

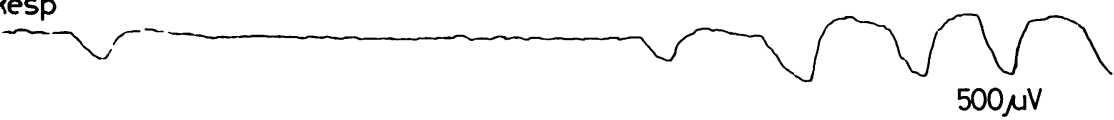

Fig. $3 E E G\left(P_{3}-A_{1}\right)$, intercostal EMG (arrows indicate inspiratory activity), and Resp (abdominal respiration; downward deflection indicates inspiration) in case 2 at $30^{\circ}$ upward tilt. Timer: 1 second. Note apnoeic period of 19 seconds.
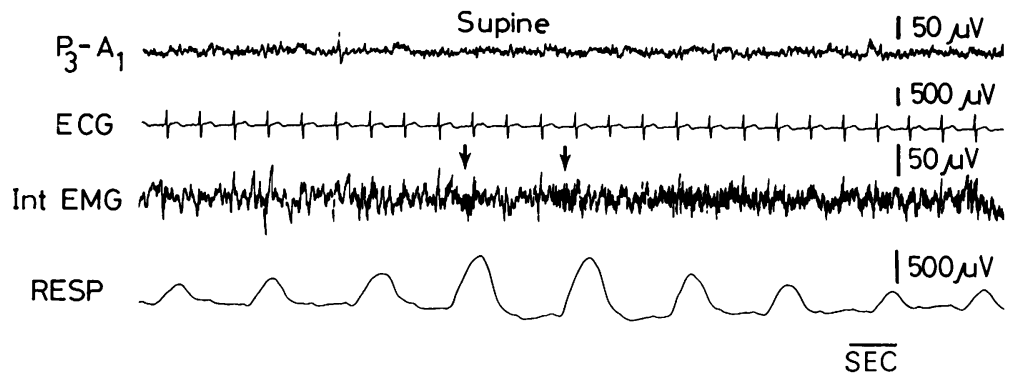

Erect

$P_{3}-A_{1}$

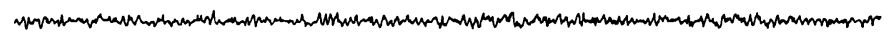

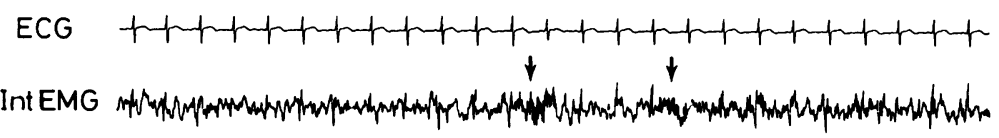

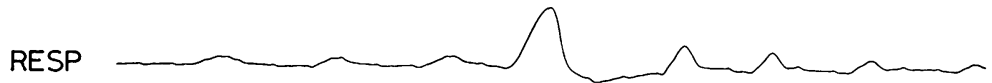

Fig. 4 Cheyne-Stokes

respiration in supine and erect positions in case 4 . EEG

$\left(P_{3}-A_{1}\right)$, intercostal EMG

(arrow points to muscle action potentials during inspiration), Resp (abdominal respiration; upward deflection indicates inspiration).

Table Pulmonary function data in supine and erect position $\left(30^{\circ}\right.$ tilt $)$ in three cases of Shy-Drager syndrome

\begin{tabular}{|c|c|c|c|c|c|c|}
\hline \multirow[b]{2}{*}{ Pulmonary function data } & \multicolumn{2}{|l|}{ Case 1} & \multicolumn{2}{|l|}{ Case 2} & \multicolumn{2}{|l|}{ Case 4} \\
\hline & Supine & Erect & Supine & Erect & Supine & Erect \\
\hline Mean respiratory rate & 25 & 21 & - & - & 24 & 20 \\
\hline Mean duration of apnoea (s) & & 10 & & 10 & & 14 \\
\hline Mean tidal volume $(\mathrm{ml})$ & 444 & 567 & 520 & 647 & 325 & 348 \\
\hline Minute ventilation $(1 / \mathrm{min})$ while breathing room air & 11.1 & 11.9 & - & - & 7.8 & 6.9 \\
\hline Minute ventilation $(1 / \mathrm{min})$ while breathing $\mathrm{CO}_{2}$ & 24.4 & 40 & - & - & 13.6 & 17.2 \\
\hline $\mathrm{PaO}_{2}$ (torr) & 65 & 65 & 70 & 70 & 60 & 74 \\
\hline \multirow{2}{*}{$\mathrm{PaCO}_{2}$ (torr) $\left\{\begin{array}{l}\text { Breathing room air } \\
\text { Breathing } \mathrm{CO}_{2}\end{array}\right.$} & 41 & 39 & 49 & 49 & 45 & 36 \\
\hline & 55 & 58 & - & - & 47 & 44 \\
\hline $\begin{array}{l}\text { Change in minute ventilation/change in } \mathrm{PaCO}_{2} \text { after } \\
\text { breathing } \mathrm{CO}_{2}\end{array}$ & 0.95 & 1.48 & - & - & 2.9 & 1.29 \\
\hline
\end{tabular}

$-=$ not measured 
position. There was diminished ventilatory response to $\mathrm{CO}_{2}$ (Lambersten, 1960) in the supine position in case 1 and low normal response in the erect position in cases 1 and 4 (Table and Fig. 5).

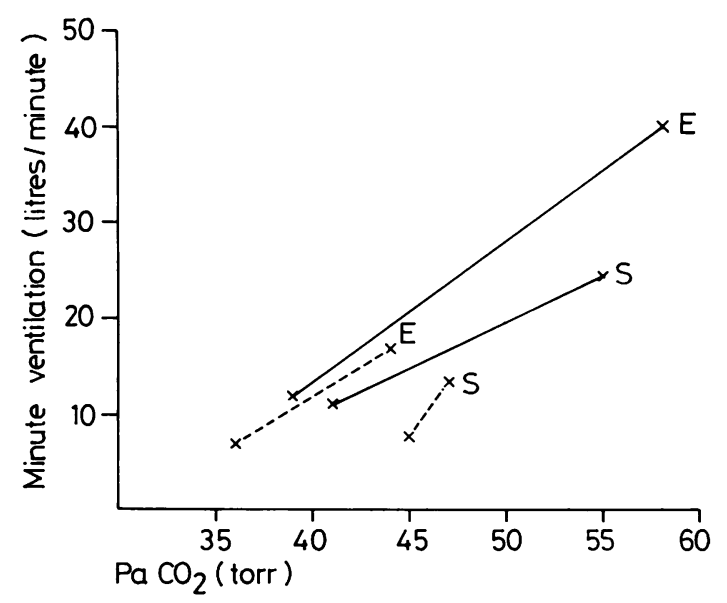

Patient $1 \quad x$

Fig. 5 Hypercapneic ventilatory response in supine and erect positions in cases 1 and 4.

\section{Discussion}

The presence of severe orthostatic hypotension accompanied by pyramidal tract, extrapyramidal, and cerebellar dysfunction in varying combinations, laboratory evidence of autonomic deficits (Chokroverty et al., 1969), and necropsy findings of striatonigral degeneration (case 1) and olivopontocerebellar atrophy (case 2) are consistent with a diagnosis of the Shy-Drager variant of primary hypotension (Shy and Drager, 1960).

In all patients, polygraphic study on a tilt-table demonstrated the occurrence of dysrhythmic breathing characterised by periodically recurring apnoea, or ineffective breathing followed by periods of normal respiration in the erect position. This type of breathing is different from the Cheyne-Stokes pattern of gradually increasing followed by gradually decreasing tidal volume. Although an occasional period of apnoea of up to 10 seconds may be noted in normal individuals, several apnoeic episodes each lasting for 8-22 seconds during brief recording in the erect position are rather unusual. In addition, the normal response of respiration to the stress of tilting upward is an increase in the rate and depth of breathing (Turner, 1927; Hamilton et al., 1932; Main, 1937;
McMichael, 1938; Franseen and Hellebrandt, 1943), although Franseen and Hellebrandt (1943) described two normal adult women who exhibited "a periodicity in amplitude occasionally so marked as to resemble Cheyne-Stokes respiration". The type of breathing in our patients resembled that noted in pontomedullary disease (Plum, 1970). The mildly reduced ventilatory response to inhalation of $\mathrm{CO}_{2}$ in case 1 (Fig. 5) would also suggest that the periodic breathing in this patient was related to dysfunction of the respiratory neurones in the brainstem tegmentum. Necropsy findings of neuronal loss and astrocytosis in the pontine tegmentum (Fig. 1) supported this assumption. We would further suggest that in the erect posture, brainstem ischaemia resulting from marked hypotension failed to stimulate the dysfunctional respiratory neurones adequately. The ventilatory dysrhythmia in a later study of our case 4 resembled Cheyne-Stokes respiration both in supine and erect positions. Similar Cheyne-Stokes breathing was noted terminally in a patient by Bannister and Oppenheimer (1972).

Eisele et al. (1971) demonstrated lack of hypoxic respiratory drive and diminished hypercapneic ventilatory response in a case of acquired dysautonomia caused by amyloidosis. In one patient with the Shy-Drager syndrome, Lockwood (1976) described cluster breathing, awake and asleep, similar to that noted in patients with bulbar poliomyelitis and brainstem infarcts. He suggested that the damage to the pontine and medullary reticular formation in his patient was responsible for the cluster breathing. Bannister and Oppenheimer (1972) reported periodic inspiratory gasps resembling apneustic breathing in two patients with Shy-Drager syndrome. Guilleminault et al. (1977) and Israel and Marino (1977) described upper airway sleep apnoea associated with vocal cord paresis in Shy-Drager syndrome. None of our patients had any evidence of palatopharyngolaryngeal paresis.

In conclusion, although the periodically recurring respiratory dysrhythmia in the erect position may not be specific for the Shy-Drager syndrome, to our knowledge this type of breathing abnormality in the erect posture has not been described previously in this entity. Furthermore, we did not observe periodic apnoea in the upright posture on a tilt-table in nine patients with another chronic neurodegenerative disease, familial and sporadic spinocerebellar degeneration (unpublished observations), four of whom had normal Valsalva responses but mild orthostatic hypotension on tilting the table upward (Chokroverty and Sachdeo, 1978). 
Note added in proof Since submitting the manuscript we have found another report of respiratory abnormality in Shy-Drager syndrome (Castaigne et al., 1977). The authors described a 65 year old man who had alveolar hypoventilation of central origin, arrhythmic respiration, many episodes of apnoea, and probable apneustic breathing four years after the onset of the disease. Necropsy findings included degenerative lesions in the substantia nigra, pontine nuclei, locus coeruleus, and intermediolateral column of the spinal cord.

\section{References}

Bannister, R., and Oppenheimer, D. (1972). Degenerative diseases of the nervous system associated with autonomic failure. Brain, 95, 457-474.

Brown, H. W., and Plum, F. (1961). The neurologic basis of Cheyne-Stokes respiration. American Journal of Medicine, 30, 849-860.

Castaigne, P., Laplane, D., Autret, A., Bousser, M. G., Gray, F., and Baron, J. C. (1977). Syndrome de Shy et Drager avec troubles du rhythme respiratoire et de la vigilance. Revue Neurologique, 133, 455-456.

Chokroverty, S., Barron, K. D., Katz, F. H., Del Greco, F., and Sharp, J. T. (1969). The syndrome of primary orthostatic hypotension. Brain, 92, 743768.

Chokroverty, S., and Sachdeo, R. (1978). Autonomic deficits in spinocerebellar degeneration. Neurology (Minneapolis), 28, 403.

Eisele, J. H., Gross, C. E., Rausch, D. C., Kurpershoek, C. J., and Zelis, R. E. (1971). Abnormal respiratory control in acquired dysautonomia. New England Journal of Medicine, 285, 366-368.

Franseen, E. B., and Hellebrandt, F. A. (1943). Postural changes in respiration. American Journal of Physiology, 138, 364-369.
Guilleminault, C., Tilkian, A., Lehrman, K., Forno, L., and Dement, W. C. (1977). Sleep apnoea syndrome: status of sleep and autonomic dysfunction. Journal of Neurology, Neurosurgery, and Psychiatry, 40, 718-724.

Hamilton, J. E., Lichty, J. S., and Pitts, W. R. (1932). Cardiovascular response of healthy young men to postural variations at varied temperatures. American Journal of Physiology, 100, 383-393.

Israel, R. H., and Marino, J. M. (1977). Upper airway obstruction in the Shy-Drager Syndrome. Annals of Neurology, 2, 83.

Lambersten, C. J. (1960). Carbon dioxide and respirations in acid-base homeostasis. Anesthesiology, 21, 642-651.

Lockwood, A. H. (1976). Shy-Drager syndrome with abnormal respirations and antidiuretic hormone release. Archives of Neurology (Chicago), 33, 292295.

Main, R. J. (1937). Alterations of alveolar $\mathrm{CO}_{2}$ in man accompanying postural change. American Journal of Physiology, 118, 435-440.

McMichael, J. (1938). Postural changes in cardiac output and respiration in man. Quarterly Journ of Experimental Physiology, 27, 55-72.

Plum, F. (1970). Neurological integration of behaviorat and metabolic control of breathing. In Breathing Hering-Breuer Centenary Symposium, pp. 159-181융 Edited by R. Porter. J. and A. Churchill: London.

Shy, G. M., and Drager, G. A. (1960). A neurologicap syndrome associated with orthostatic hypotension 2 Archives of Neurology (Chicago), 2, 511-527.

Turner, A. H. (1927). The circulatory minute volumes of healthy young women in reclining, sitting an standing positions. American Journal of Physiologed 80, 601-630.

Verel, D. (1951). Postural hypotension: the localisation of the lesion. British Heart Journal, 13, 61-67. 